\title{
Risk of Development of Resistance in Patients with Non-Cystic Fibrosis Bronchiectasis Treated with Inhaled Antibiotics
}

\author{
Kate H. Regan ${ }^{1,2} \cdot$ Adam T. Hill ${ }^{1,2}$
}

Published online: 5 July 2018

(C) The Author(s) 2018

\begin{abstract}
Purpose of Review Bronchiectasis is a debilitating chronic lung disease characterised by recurrent bacterial infection and colonisation with significant associated morbidity and mortality. To date, there are few licenced treatments, and the mainstay of clinical management is prompt antibiotic therapy for exacerbations and regular airway clearance. Inhaled antibiotics are a potential long-term treatment for those with recurrent exacerbations, and represent an obvious advantage over other routes of administration as they achieve high concentrations at the site of infection whilst minimising systemic side effects. The main caveat to such treatment is the development of antimicrobial resistance due to altered selection pressures.

Recent Findings Numerous studies of various inhaled antimicrobials have demonstrated favourable safety and efficacy profiles for bronchiectasis patients with chronic infection, which are supportive of their use in clinical practice.

Summary There is no convincing evidence of treatment-emergent pathogens or pathogens developing resistance to the inhaled antibiotic therapy.
\end{abstract}

Keywords Non-CF bronchiectasis $\cdot$ Inhaled antibiotics $\cdot$ Antibiotic resistance

\section{Introduction}

Bronchiectasis is a chronic lung disease characterised by permanent dilatation of the lower airways, resulting in a clinical syndrome of chronic productive cough and recurrent lower respiratory tract infections $[1,2]$. Approximately $2-5$ per 1000 population are affected by this debilitating lung disease, with incidence highest in patients aged $>60[3,4]$. Bronchiectasis represents the common pathological endpoint of a variety of underlying conditions, including cystic fibrosis (CF). Bronchiectasis is generally categorised as either $\mathrm{CF}$-related or non-CF-related bronchiectasis (NCFB), the latter of which will form the focus of this review. NCFB most frequently occurs following a severe lower respiratory tract infection or is

This article is part of the Topical Collection on Bronchiectasis

Kate H. Regan

KateRegan4@gmail.com

1 The Queen's Medical Research Institute, University of Edinburgh/ MRC Centre for Inflammation Research, Edinburgh, UK

2 Department of Respiratory Medicine, Royal Infirmary of Edinburgh, Edinburgh, UK idiopathic, though there are numerous potential aetiologies including allergic bronchopulmonary aspergillosis (ABPA), immune function disorders and ciliary dyskinesias [5]. The gold standard technique for the diagnosis of bronchiectasis is high resolution CT chest, which demonstrates bronchial dilatation to greater than the diameter of the accompanying pulmonary artery and a failure of small airways tapering [6॰]. The resulting impairment in mucociliary clearance (MCC) leads to excess secretions in the lower airways, providing an ideal environment for bacterial infection to thrive [7].

In the early phases of the disease, infection is cleared by prolonged courses of oral or intravenous antibiotics. Over time, the cycle of recurrent infections progresses to bacterial colonisation in the majority of patients, leading to a progressive decline in lung function, worsening quality of life and increasing lung damage. Unlike in $\mathrm{CF}$, where bacterial colonisation tends to follow a 'classical' pattern of early Staphylococcus aureus (SA) infection and almost inevitable later colonisation with Pseudomonas aeruginosa (PA), the chronic pathogens implicated NCFB are more heterogenous [8, 9]. The most common chronic infections in NCFB are Haemophilus influenzae (HI) and Pseudomonas aeruginosa (PA), with Staphylococcus aureus (SA), Streptococcus pneumoniae (SP), Moraxella catarrhalis (MC) and enteric gram-negative organisms also 
commonly cultured from lower respiratory tract secretions $[10$, 11]. In recent years, there has also been increasing focus on the role of non-tuberculous mycobacterial (NTM) infection, which is now known to affect up to $10 \%$ of the bronchiectasis population $[12,13]$. Once established in the lower airway, bacteria become extremely difficult to eradicate from the lung.

Patients with bronchiectasis report poor health-related quality of life (HRQoL), significant fatigue and a high burden of respiratory symptoms, which are closely linked to bacterial load in the lower airway and the resultant respiratory and systemic inflammation [14-20]. Despite this, there remain few recommendations for their long-term management, with treatment focussed on rapid initiation of culture-targeted antibiotics for respiratory exacerbations and daily airway clearance. Given the role of bacterial infection in the severity of symptoms and disease progression, there has been much focus on the role of long-term antibiotic therapy in NCFB.

Inhaled antibiotics provide an obvious advantage over enterally or parenterally administered antibiotics, as they reach much higher concentrations at the site of infection whilst remaining at low levels in the systemic circulation, thereby reducing potential side effects of long-term use such as renal, hepatic or auditory dysfunction. Inhaled antibiotics have long been studied and licenced in the CF population, where long-term use of inhaled anti-pseudomonal antibiotics such as colistin and tobramycin have demonstrated excellent clinical efficacy in reducing lung function decline, frequency of exacerbations and delaying chronic infection [21-23]. Disappointingly to date, few high-quality trials of inhaled antibiotics in NCFB have been published, and no inhaled antimicrobial preparations are currently licenced for use in this patient group. Despite this, current guidelines recommend the off-label use of inhaled antibiotics in the management of patients with chronic lung infection, and use of nebulised colistin and gentamicin is common in clinical practice [6•,24]. According to data from the most recent British Thoracic Society bronchiectasis audit, some $10 \%$ of British bronchiectasis patients were receiving long-term inhaled antimicrobial therapy, of whom $86 \%$ received colistin, $6 \%$ gentamicin, $4 \%$ tobramycin and $4 \%$ others [4].

One of the main, and understandable, concerns of using long-term inhaled antibiotics as a suppressive therapy in both $\mathrm{CF}$ and NCFB is the emergence of resistant bacterial strains, further complicating the management of an already extremely challenging group of patients. Traditionally, we have assumed that the chronic exposure of a pathogen to a level of a specific antimicrobial that is insufficient to eradicate that pathogen will lead to the development of resistant strains via altered selection pressures. However, it is difficult to know whether established minimum inhibitory concentrations (MICs), developed in conjunction with parenteral break points, are applicable when the airway concentration of inhaled antibiotics significantly exceeds that which can be safely be achieved via the parenteral route due to the risk of systemic toxicity [25-27]. Furthermore, the MIC standards used by studies vary according to their geographical location, with most European centres ascribing to EUCAST standards, and those in the USA utilising the CLSI breakpoints [28, 29].

To date, most inhaled antibiotic studies have focused on patients colonised with PA, likely since numerous studies have independently linked this with increased morbidity and mortality, and because its management has been so extensively studied in CF [17, 30-32]. Chronic PA infection affects approximately $20 \%$ of NCFB patients, but the role of colonisation with other pathogens in disease course is less well defined $[33,34]$. In this review article, we will focus on the risk of developing resistance in those taking long-term inhaled antibiotic therapy for NCFB.

\section{Tobramycin}

\section{Couch [35]}

This randomised placebo-controlled trial used tobramycin solution for inhalation (TSI) (TOBI®) for 4 weeks in patients colonised with PA. Seventy-four patients were enrolled and were equally divided between the treatment ( $300 \mathrm{mg}$ TSI BD for 28 days) and placebo (1.25 mg quinine sulphate) arms, with the primary outcome being a reduction in sputum PA density. The study demonstrated a significant reduction in sputum bacterial load in the treatment group, with a mean reduction in PA bacterial load of $4.8 \log _{10}$ and $4.54 \log _{10}$ at 2 and 4 weeks respectively. Some regrowth was noted 2 weeks following discontinuation of therapy. At enrolment, all patients had tobramycin sensitive PA. 3/36 (8\%) treated and 1/ $34(3 \%)$ placebo patients developed PA isolates with tobramycin MICs $\geq 16 \mu \mathrm{g} / \mathrm{mL}$ (tobramycin parenteral breakpoint) during the study. The duration of follow-up was insufficient to determine whether these strains remained present following study discontinuation. There was no discussion of emergence of opportunistic bacterial species, though the effect of treatment on co-colonising pathogens was reviewed. Resistance was not tested for these groups.

\section{Scheinberg et al. [36]}

This open-label, uncontrolled study of $300 \mathrm{mg}$ BD of TSI consisted of $3 \times 2$-week on/off treatment cycles and a 40-week follow-up period via review of medical notes. Forty-one patients with severe bronchiectasis colonised with PA were enrolled in this trial, whose primary outcome measure was improvement HRQoL. They demonstrated a mean reduction of 1.5 units in mean pulmonary total symptom severity score and 9.8 units in the St. George's respiratory questionnaire (SGRQ) [37]. They reported an eradication rate of 
22.2\%; quantitative sputum bacteriology was not reported. Two (5\%) of the study participants developed PA isolates with tobramycin MICs $\geq 16 \mu \mathrm{g} / \mathrm{mL}$ ( 16 and $128 \mu \mathrm{g} / \mathrm{mL}$ ) at the end of treatment. No further resistance data following the end of the treatment period is reported.

\section{Orriols et al. 1999 [38]}

In this early study, Orriols et al. conducted an open-label 12-month trial of nebulised ceftazidime $1000 \mathrm{mg} \mathrm{BD}$ and tobramycin $100 \mathrm{mg}$ BD vs. standard care. They recruited patients with chronic PA infection who had a 2-year history of frequent exacerbations and difficult to control symptoms. Following a 2-week course of intravenous ceftazidime and tobramycin, patients were randomly allocated to receive treatment $(n=7)$ or standard care $(n=8)$. During the course of the trial, patients admitted with an exacerbation who subsequently had sputum cultures that demonstrated resistance were converted to piperacillin (100 $\mathrm{mg} \mathrm{BD}$ ) in place of ceftazidime and amikacin (100 mg BD) in place of tobramycin for the remainder of the study. The primary outcome measure was the number and duration of hospital admissions. Patients in the treatment arm had significantly fewer admissions; mean $( \pm$ SEM) $0.6( \pm 1.5)$ vs. $2.5( \pm 2.1)$ and a shorter duration of admission, $13.1( \pm 34.8)$ days and $57.9( \pm 41.8)$ days respectively than those in the standard care group. There was no evidence of emergence of new opportunistic pathogens within either group and no successful eradication, though two treated and one untreated patient had transiently negative cultures. Of the treated group, one patient developed a tobramycin-resistant PA isolate without clinical worsening. In the group receiving standard care, four patients developed in vitro resistance to ceftazidime, one of whom also isolated tobramycin-resistant PA necessitating a change in antibiotic treatment. Overall, the incidence of resistant isolates was therefore higher in the group not receiving inhaled antibiotics, who had more admissions and thus more exposure to intravenous antibiotics.

\section{Barker et al. [39]}

Barker and colleagues conducted a 4-week double-blinded RCT of $300 \mathrm{mg}$ BD of nebulised TSI (TOBI $®$ ) vs. placebo (1.25 mg quinine sulphate), who were reviewed at $0,2,4$ and 6 weeks ( 2 weeks post completion of treatment). Clinically stable patients with a history of chronic PA infection who could produce a sputum sample with at least $10^{4} \mathrm{CFU} / \mathrm{g}$ PA were recruited. The primary outcome measure was a reduction in sputum PA bacterial density. They demonstrated a significant reduction in sputum bacterial load at all time points; at week 4 , the reduction was $4.54 \log _{10} \mathrm{CFU} / \mathrm{g}$ vs. no change in the placebo group. At week 6, eradication was achieved in $35 \%$ of treated vs. $0 \%$ of placebo. Twenty-six percent of treated and $14 \%$ of the placebo group had a PA isolate at week 6 that had $\geq 4$-fold increase in MIC vs. baseline. However, only $11 \%$ treated vs. $3 \%$ placebo had isolates with a MIC high enough to be considered resistant ( $\geq 16 \mu \mathrm{g} / \mathrm{mL}$ ) [29]. There was no discussion of treatment-emergent pathogens.

\section{Drobnic et al. [40]}

Drobnic et al. recruited patients chronically colonised with PA. This double blind, placebo-controlled crossover trial consisted of two arms of twice-daily nebulised tobramycin $300 \mathrm{mg}$ or $0.9 \% \mathrm{NaCl}$ (placebo) for 6 months, with a 1 month washout period between. Thirty patients chronically colonised with PA received 2 weeks of intravenous ceftazidime and tobramycin to achieve clinical stability prior to the initiation of nebulised therapy. Patients with PA isolates resistant to tobramycin (zone diameter $\geq 8 \mathrm{~mm}$ with a $0.01 \mathrm{~mm}$ tobramycin disk) were excluded from the trial. Primary endpoints were the number and duration of hospital admissions. Secondary endpoints included pulmonary function, days of antibiotic treatment, quality of life, sputum bacterial load, evidence of resistant strains and emergence of opportunistic bacteria. The trial demonstrated a significant reduction in the number mean, \pm SD $0.15 \pm 0.37$ vs. $0.75 \pm 1.16$ and duration of admissions, $2.05 \pm 5.03$ days vs. $12.65 \pm 21.8$ days in the tobramycin and placebo arms respectively. There was also a non-significant trend towards decreased number of days of antibiotic therapy. There was no evidence of effect on pulmonary function, quality of life or frequency of exacerbations. Emergence of tobramycin-resistant PA was seen in two patients during the treatment arm and two patients during the second arm of placebo treatment. Two months following the end of the treatment period, all patients had fully susceptible PA (MIC $<8 \mu \mathrm{g} / \mathrm{mL}$ ) in their sputum. Serial cultures demonstrated the emergence of Alcaligenes xylosoxidans and $S$. pneumoniae in two patients during the treatment arm and Acinetobacter species and Stenotrophomonas maltophilia during the placebo arm in two others. Overall, there was no difference in the emergence of bacterial resistance or acquisition of new opportunistic pathogens in response to treatment over the study duration.

\section{Orriols et al. 2015 [41]}

Similarly to the above trials, Orriols et al. conducted a double-blinded RCT of $300 \mathrm{mg}$ BD nebulised TSI (TOBI®) over 3 months in patients with first isolation of PA in sputum. Experimental treatment was initiated following a 2-week course of intravenous ceftazidime and tobramycin within 4 weeks of PA detection. Due to the high rates of tobramycin-associated bronchospasm reported in numerous previous trials of both $\mathrm{CF}$ and NCFB, patients also received a short-acting bronchodilator $1 \mathrm{~h}$ prior to treatment. The primary outcome measure was the proportion of PA-free 
patients; at the end of month $1,90.9 \%$ of treated vs. $76.5 \%$ of placebo had negative sputum cultures, compared with 54.5 and $29.4 \%$ respectively at the end of the study. In addition, as found by Drobnic et al., the trial demonstrated a significant reduction in the number and length of admissions, and additionally reported a significant increase in the number of patients with PA negative cultures at the end of the study period. There was no improvement in pulmonary function tests, consistent with other trials, and no evidence of systemic toxicity. No tobramycin-resistant isolates of PA were detected at any time point during the course of the study. Opportunistic pathogens, including S. maltophilia and A. xylosoxidans, were detected in two treated and six placebo patients, suggesting no trend towards treatment-emergent pathogens.

\section{Ciprofloxacin}

\section{ORBIT-2 [42]}

ORBIT-2 was a multi-centre RCT examining the effect of a twice-daily liposomal ciprofloxacin DPI vs. placebo over a 6-month period in 28-day on/off cycles. They recruited 42 subjects with a history of chronic ciprofloxacin-sensitive PA infection with at least 2 exacerbations in the preceding year. The primary outcome measure was a reduction in sputum bacterial load at the end of the first 28-day treatment cycle, with mean (SD) 4.2 (3.7) vs. -0.08 (3.8) $\log _{10}$ CFU/g reduction in treated vs. placebo. This persisted to 84 days post treatment initiation, and 60 vs. $14 \%$ had successful PA eradication at 28 days. They also demonstrated a significant increase in time to first pulmonary exacerbation, though there was no significant effect on HRQoL or pulmonary function. Emergence of new opportunistic sputum pathogens was seen in $55 \%$ of placebo and $45 \%$ of treated patients, with the most common new isolate being S. maltophilia. Ciprofloxacin resistance was determined according to CLSI breakpoints; $38 \%$ of placebo patients isolated an intermediately sensitive or resistant PA isolate at any time point vs. $50 \%$ of treated subjects. The change in MIC of the most resistant isolates identified at 28 days did not differ significantly between the two groups.

\section{Wilson et al. [43]}

This phase II multi-centre RCT explored the use of BD ciprofloxacin $32.5 \mathrm{mg}$ DPI over 4 weeks in the management of adult bronchiectasis patients with chronic airway pathogens who were frequent exacerbators $(\geq 2$ per year). Subjects who cultured PA, SA, SP, HI, MC, S. maltophilia, Achromobacter xylosoxidans or Enterobacteriaceae were eligible for study entry. The primary endpoint was a reduction in sputum bacterial load at the end of treatment (day 28), and the mean (range) reductions were $3.62 \log _{10}(9.78-5.02) \mathrm{CFU} / \mathrm{g}$ in the active arm vs. 0.27 (7.95-5.25) $\log _{10} \mathrm{CFU} / \mathrm{g}$ in the placebo arm. There was also a non-significant trend towards reduced bacterial load at follow-up days 42 and 56 before a return to baseline at the end of study day 84 . At day 8 , pathogen eradication was achieved in $48 \%$ treated vs. $12 \%$ of the placebo group. Twelve treated and 24 placebo patients had sputum cultures positive for alternative respiratory pathogens vs. baseline; S. maltophilia was the most frequent new isolate. Increases in bacterial MIC to $>4 \mathrm{mg} / \mathrm{L}$ (ciprofloxacin parenteral break point) were reported in six treated and zero placebo patients; of these isolates only one still had an elevated MIC at the end of the study.

\section{RESPIRE I and II [44•, 45•]}

The RESPIRE trials were multi-centre double-blinded RCTs conducted over 48 weeks; consisting of treatment with ciprofloxacin DPI $32.5 \mathrm{mg}$ BD in either 14- or 28-day on/off cycles vs. placebo. Recruited patients had idiopathic or post-infective bronchiectasis and a history of recurrent exacerbations. All were chronically colonised with PA, HI, MC, SA, SP, SM or Burkholderia cepacia complex at baseline. Each trial recruited over 400 patients with high completion rates and study drug compliance $>90 \%$ in all groups. RESPIRE I demonstrated that ciprofloxacin DPI administered in 14-day on/off cycles significantly reduced the exacerbation rate by $39 \%$ over 48 weeks (mean number of exacerbations 0.6 vs. 1.0 treatment vs. pooled placebo), along with a significant increase in pathogen eradication and improvement in HRQoL as assessed by the SGRQ. The outcomes for the 28-day on/off cycles were not statistically significant. In RESPIRE II, there was a minor trend towards prolonged time to the next exacerbation but the overall exacerbation rate in participants was low, which was believed to play a role in the lack of significant evidence of treatment effect. The RESPIRE I trial reported that $24.5 \%$ of their patients demonstrated an elevated ciprofloxacin MIC at baseline. Interestingly, subgroup analyses did not demonstrate reduced efficacy in these patients, further supporting the hypothesis that the higher antimicrobial concentrations achieved in the lung following the use of inhaled antibiotics renders MIC data based on parenteral breakpoints irrelevant. Across the course of the study, $20.4 \%$ of the 14 -day, $9.2 \%$ of the 28 -day and $12.3 \%$ of the pooled placebo arms demonstrated an elevated MIC (relative to the CLSI breakpoint standards) in at least one isolate at any time point. At the end of the study visit ( 8 weeks post treatment cessation), $6.3 \%$ of participants had newly resistant isolates-7.3, 9.2 and $2.2 \%$ of the 14,28 and placebo arms respectively. Results from RESPIRE II were similar. The authors postulate that any treatment-related MIC increase is likely to be offset by the overall decrease in bacterial load, reduced exacerbation rate and consequent reduction in systemic antimicrobial therapy. 


\section{Colistin}

\section{Haworth et al. [46]}

Despite the fact that colistin is the most frequently used off-label inhaled antibiotic in the management of chronic bronchial infection in NCFB, the trial by Haworth et al. is the only double-blinded RCT examining its role in this population. The bulk of the evidence supporting its use in the management of PA colonisation is extrapolated from the CF world, where it has been a mainstay of treatment for several decades. Haworth's group conducted a trial of 6 months duration exploring the use of 1 million IU BD of nebulised colistin vs. placebo $(0.45 \% \mathrm{NaCl})$ in adult bronchiectasis patients with a history of chronic PA infection following a course of anti-pseudomonal antibiotics for management of a pulmonary exacerbation. Their primary endpoint was time to the first exacerbation, and time to the first exacerbation based on adherence to study drug was amongst their secondary end points. Forty-nine percent of patients in the colistin group vs. $59 \%$ in the placebo group experienced an exacerbation, with median time to exacerbation 165 vs. 111 days respectively, which was not statistically significant. However, patients with a compliance of $\geq 80 \%$ to study medication had a significant increase in time to first exacerbation, with $35 \%$ of treated and $82 \%$ of placebo patients experiencing an exacerbation; median time to the first exacerbation could not be calculated in the colistin group due to the number of patients completing the treatment course. They also demonstrated improved HRQoL (SGRQ) with no effect on pulmonary function. No colistin-resistant PA isolates (as per EUCAST guidelines) were identified at any time point during the study and there was no difference between the emergent new pathogens between the treated and untreated groups.

\section{Gentamicin}

\section{Murray et al. [47]}

Following colistin, nebulised gentamicin is the next most frequently used off-label nebulised antibiotic in the management of NCFB complicated by chronic bronchial infection. The rationale behind the use of gentamicin is its broad spectrum of action, but its use intravenously confers a cumulative risk of nephro- and ototoxicity. In this single-blinded RCT, NCFB patients with chronic lung infection with any potential respiratory pathogen and frequent ( $\geq 2$ /year) exacerbations were randomised to receive $80 \mathrm{mg} \mathrm{BD}$ of nebulised gentamicin or $0.9 \% \mathrm{NaCl}$ over 12 months and were reviewed at 3-monthly intervals. The primary end points were sputum bacterial density and pathogen eradication. The trial reported a significant reduction in sputum bacterial load at 12 months in the gentamicin-treated group, with median bacterial density (range) $2.96(1.0-5.9) \log _{10} \mathrm{CFU} / \mathrm{mL}$ in the gentamicin treated group vs. 7.67 (7.34-8.17) $\log _{10} \mathrm{CFU} / \mathrm{mL}$ in the placebo group. $30.8 \%$ of PA-colonised patients and $92.8 \%$ of those colonised with other pathogens had negative sputum cultures at the end of treatment with gentamicin. In addition, the treatment group reported significantly increased exercise tolerance, HRQoL (SGRQ), fewer exacerbations, increased time to first exacerbation and decreased sputum inflammatory indices during treatment. At 3-month post treatment, cessation indices had returned to baseline, highlighting that continuous treatment is likely to be required for sustained clinical improvement. As with other trials, there was no effect demonstrated on pulmonary function, and no evidence of systemic toxicity. No gentamicin-resistant or intermediate resistant isolates of PA or enteric gram-negative organisms were isolated at baseline, end of treatment or end of study. Other pathogens were not tested for resistance. Furthermore, there was no evidence of new treatment-emergent pathogens.

\section{Aztreonam}

\section{AIR-BX1 and 2 [48]}

The AIR-BX trials were identical multi-centre randomised controlled trials of aztreonam lysine solution for inhalation (AZLI), an inhaled anti-pseudomonal antibiotic with a strong evidence base in the management of chronic airway infection in the CF population [49-51]. Patients chronically colonised with gram-negative organisms, (other than $H$. influenzae alone), and a history of chronic sputum production were recruited to receive two cycles of 4 weeks of nebulised AZLI $75 \mathrm{mg}$ TDS or matched placebo followed by 4 weeks off treatment. The primary endpoint was change from baseline in quality of life bronchiectasis respiratory symptoms score (QOL-B-RSS) at 4 weeks [52, 53]. In AIR-BX1, 134 were randomised to AZLI and 132 to placebo; in AIR-BX2, 136 received AZLI and 138 placebo. The mean adherence to study medication was high in both trials across treatment allocations. In AIR-BX1, following 4 weeks of treatment, there was a mean (SE) increase in QOL-B-RSS of 6.4 (1.4) points in the AZLI group vs. 5.6 (1.4) points in the placebo group. Similarly at 12 weeks, the mean (SE) increase was 5.7 (1.6) points vs. 4.4 (1.5) points for the AZLI and placebo groups respectively, with neither time point demonstrating statistical significance. In AIR-BX2, the mean (SE) increase was 7.9 (1.3) points for the AZLI and 3.3 (1.3) points for the placebo groups at 4 weeks, and 5.3 (1.4) points vs. 4.1 (1.4) points for the AZLI and placebo groups at 12 weeks. At 4 weeks, the mean increase between the AZLI and placebo groups of 4.6 points was statistically significant $(p=0.011)$; however, the minimum clinically important difference validated for QOL-B 
is 8 points, and as such, this result was not deemed to be clinically relevant [54]. The proportion of patients with detectable organisms in sputum was reduced in the AZLI-treated groups after baseline, with AZLI also demonstrating decreased sputum bacterial load. In AIR-BX1, $15 \%$ of AZLI-treated vs. $6 \%$ of placebo-treated patients with detectable organisms in sputum at 4 weeks demonstrated increases in aztreonam MIC of 4-fold or greater. Similarly at 12 and 16 weeks respectively, 35 and $23 \%$ of AZLI and 11 and $14 \%$ of placebo isolates demonstrated MIC increase of $\geq$ 4-fold. In AIR-BX2, at 4, 12 and 16 weeks respectively, increases in MIC of $\geq 4$-fold were seen in 23, 34 and $20 \%$ of those receiving AZLI vs. 7, 11 and $6 \%$ of the placebo group. Actual MIC values for the organisms were not reported in the study; therefore, it is difficult to determine whether these increases correspond with clinically significant resistance. There was no discussion of treatment-emergent pathogens.

\section{Discussion}

Aside from the tolerability of inhaled antibiotics, the main clinical concern with their long-term use in chronic bronchial infection is the development of resistant bacterial strains. There has been much media focus on the so-called antibiotic apocalypse and the imminent arrival of the 'post-antibiotic era'. Given the challenges modern healthcare faces in the form of MDR infections such as MRSA, vancomycin-resistant enterococci and Clostridium difficile infection, which have arisen largely due to historical over-prescription of antibiotic therapy, it is perhaps unsurprising that such concerns are at the forefront of most clinician's minds. Furthermore, the CF and NCFB populations are unique in their pathophysiology with respect to the fact that these infections may need suppressive therapy over several decades; which has been hitherto uncharted territory in terms of antibiotic prescribing. Nonetheless, observational studies and clinical trials have repeatedly demonstrated the correlation between worse clinical outcomes and bacterial load, and as such suppressive treatment of these chronic infections may be beneficial in the management of these patients for whom eradication is unlikely to be a realistic clinical goal $[19,20]$.

Above, we have reviewed numerous studies examining the role of inhaled antibiotic preparations in the management of NCFB, and it is clear that most studies are supportive of their role in reducing sputum bacterial load with consequent improvement in exacerbation rates, frequency and duration of hospital admissions and HRQoL [35, 36, 38-43, 44•, 45•, 46-48]. See Table 1 for a summary of findings. Reassuringly, the studies exploring the use of tobramycin, colistin and gentamicin did not demonstrate significant emergence of antimicrobial-resistant isolates in sputum and any transient increases in MIC generally returned to baseline following treatment cessation. In addition, there was little evidence that reduction in bacterial load or apparent eradication of the dominant pathogen was associated with treatment-emergent pathogens.

The reasons for this lack of resistance development are likely to be multifactorial and are yet to be fully explored. It seems likely that traditional MIC as determined in conjunction with parenteral breakpoints are unlikely to be applicable in the case of inhaled antibiotics, which lead to sputum concentrations significantly greater than that which can be safely administered parenterally without toxic effects. As the use of inhaled antibiotics is further explored, airway-adjusted MICs may be developed that correspond more directly with the likely concentrations achieved via the inhalational route. It is possible that any transient and potentially clinically insignificant increases in MIC will be offset by the benefits of reduced exacerbations and thereby exposure to systemic antimicrobial therapies.

In those trials examining the role of ciprofloxacin and aztreonam, the data regarding increases in resistance were less clear cut. Both the RESPIRE and AIR-BX trials demonstrated that a higher proportion of patients receiving active treatment developed isolates with significantly increased MIC than those receiving placebo at the end of treatment visits. In addition, even following a period off treatment, the percentage of patients with resistant isolates was still increased compared to baseline in the treatment vs. the placebo groups, though to a lesser extent than isolates taken immediately following active treatment. Interestingly, the RESPIRE trials included a sub-analysis of those patients who started the trial with ciprofloxacin-resistant PA isolates, which demonstrated no difference in treatment efficacy for these patients, underpinning the view that in vitro resistance does not necessarily transfer to lack of in vivo drug efficacy. In vitro resistance, in addition, may not be applicable as inhaled antibiotics achieve concentrations several folds higher than the MIC. Furthermore, it would be interesting to explore the relationship between these increases in MIC and the intermittent modality of drug administration utilised in these particular trials. Historically, 28-day on/off treatment cycles have been favoured in the CF population as they are thought to reduce development of bacterial resistance. However, several of the studies we have discussed above highlight the necessity of continuous therapy in maintaining clinical efficacy in the NCFB population, and perhaps this approach is required to ensure continued bacterial suppression and reduce the development of resistant isolates in NCFB.

The majority of the trials we have discussed relate only to chronic PA bronchial infection, which whilst a significant complication of late-stage NCFB is not the most common colonising airway pathogen. It is true that unlike with PA, the effect of colonisation with other potential respiratory pathogens is perhaps less definitively linked with increased 


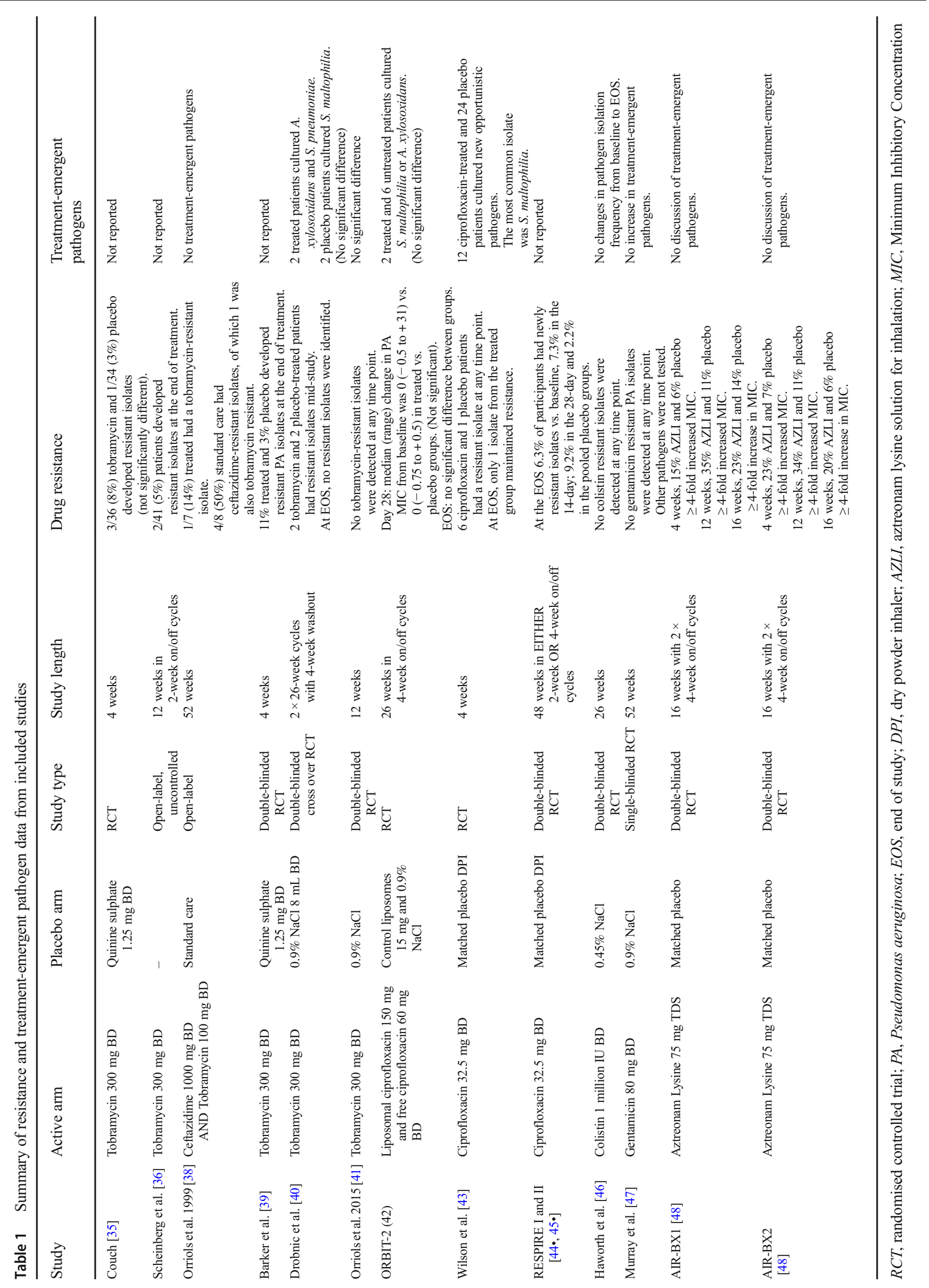


morbidity and mortality. Nonetheless, development of safe and effective inhaled treatments that can target the wide range of infecting pathogens seen in NCFB may be a useful therapeutic strategy. Few of the trials enrolling such patients have comprehensively reported MIC testing across all pathogens, but the data available are suggestive of low rates of resistant isolates.

None of the trials we have identified have explored the use of inhaled antibiotic preparations in NCFB for periods $>$ 1 year, which given the chronic nature of bronchial infection in these patients and the fact that all trials indicate the need for continuous therapy to achieve sustained clinical improvement is perhaps the biggest caveat to these conclusions. It is certainly clear that observational studies of patients on inhaled antimicrobial therapy will need to be extended over many years to truly determine their long-term impact. Furthermore, the role of intermittent vs. continuous treatment with the same inhaled antimicrobial has not been examined in any of the trials we have discussed. Exploration of this is particularly important in order to optimise therapeutic regimens to be safe, tolerable and efficacious in NCFB, whilst minimising the emergence of resistant bacterial strains.

In our opinion, the current evidence base collectively leans in favour of the benefits of inhaled antimicrobial therapy in improving patient morbidity and HRQoL, with little evidence of emerging bacterial resistance, treatment-emergent pathogens or systemic toxicity up to 1 year of therapy.

\section{Compliance with Ethical Standards}

Conflict of Interest Kate Regan declares no conflict of interest. Adam Hill reports serving an advisory board for Bayer, outside the submitted work.

Human and Animal Rights and Informed Consent This article does not contain any studies with human or animal subjects performed by any of the authors.

Open Access This article is distributed under the terms of the Creative Commons Attribution 4.0 International License (http://creativecommons. org/licenses/by/4.0/), which permits unrestricted use, distribution, and reproduction in any medium, provided you give appropriate credit to the original author(s) and the source, provide a link to the Creative Commons license, and indicate if changes were made.

\section{References}

Papers of particular interest, published recently, have been highlighted as:

- Of importance

1. King PT, Holdsworth SR, Freezer NJ, Villanueva E, Holmes PW. Characterisation of the onset and presenting clinical features of adult bronchiectasis. Respir Med. 2006;100(12):2183-9.
2. Laennec RTH, Forbes SJ. A treatise on the diseases of the chest, and on mediate auscultation. Samuel S. and William Wood; 1838. 848 p.

3. Quint JK, Millett ERC, Joshi M, Navaratnam V, Thomas SL, Hurst $\mathrm{JR}$, et al. Changes in the incidence, prevalence and mortality of bronchiectasis in the UK from 2004 to 2013: a population-based cohort study. Eur Respir J 2015 Nov 5; ERJ-01033-2015.

4. Hill AT, Routh C, Welham S. National BTS bronchiectasis audit 2012: is the quality standard being adhered to in adult secondary care? Thorax. 2014;69(3):292-4.

5. Gao Y-H, Guan W-J, Liu S-X, Wang L, Cui J-J, Chen R-C, et al. Aetiology of bronchiectasis in adults: a systematic literature review. Respirol Carlton Vic. 2016;21(8):1376-83.

6. Hill AT, Bilton, Diana, Brown, Jerry, Burns, Graham, Calvert, James, Heslop, Karen, et al. British thoracic society quality standards for clinically significant bronchiectasis in adults. British Thoracic Society Reports, Vol 4, No 1; 2012. Consensus guidelines in the management of adult bronchiectasis, summarising the current evidence base and standard practice.

7. Cole PJ. Inflammation: a two-edged sword-the model of bronchiectasis. Eur J Respir Dis Suppl. 1986;147:6-15.

8. Rajan S, Saiman L. Pulmonary infections in patients with cystic fibrosis. Semin Respir Infect. 2002;17(1):47-56.

9. Coutinho HDM, Falcão-Silva VS, Gonçalves GF. Pulmonary bacterial pathogens in cystic fibrosis patients and antibiotic therapy: a tool for the health workers. Int Arch Med. 2008;1:24.

10. Angrill J, Agustí C, Celis R de, Rañó A, Gonzalez J, Solé T, et al. Bacterial colonisation in patients with bronchiectasis: microbiological pattern and risk factors. Thorax 2002 1;57(1):15-19.

11. Alanin MC, Nielsen KG, von Buchwald C, Skov M, Aanaes K, Høiby N, Johansen HK A longitudinal study of lung bacterial pathogens in patients with primary ciliary dyskinesia. Clin Microbiol Infect 2015;21(12):1093.e1-1091093.e7.

12. Chu H, Zhao L, Xiao H, Zhang Z, Zhang J, Gui T, et al. Prevalence of nontuberculous mycobacteria in patients with bronchiectasis: a meta-analysis. Arch Med Sci AMS. 2014;10(4):661-8.

13. Fowler SJ, French J, Screaton NJ, Foweraker J, Condliffe A, Haworth CS, et al. Nontuberculous mycobacteria in bronchiectasis: prevalence and patient characteristics. Eur Respir J. 2006;28(6): 1204-10.

14. Martínez-García MA, Perpiñá-Tordera M, Román-Sánchez P, Soler-Cataluña JJ. Quality-of-life determinants in patients with clinically stable bronchiectasis. Chest. 2005;128(2):739-45.

15. Wilson CB, Jones PW, O'Leary CJ, Hansell DM, Cole PJ, Wilson R. Effect of sputum bacteriology on the quality of life of patients with bronchiectasis. Eur Respir J. 1997;10(8):1754-60.

16. Olveira C, Olveira G, Gaspar I, Dorado A, Cruz I, Soriguer F, et al. Depression and anxiety symptoms in bronchiectasis: associations with health-related quality of life. Qual Life Res. 2013;22(3):597605.

17. Araújo D, Shteinberg M, Aliberti S, Goeminne PC, Hill AT, Fardon $\mathrm{TC}$, et al. The independent contribution of Pseudomonas aeruginosa infection to long-term clinical outcomes in bronchiectasis. Eur Respir J. 2018;51(2):1701953.

18. Hester KLM, Macfarlane JG, Tedd H, Jary H, McAlinden P, Rostron L, et al. Fatigue in bronchiectasis. QJM Int J Med. 2012;105(3):235-40.

19. Hill AT, Campbell EJ, Hill SL, Bayley DL, Stockley RA. Association between airway bacterial load and markers of airway inflammation in patients with stable chronic bronchitis. Am J Med. 2000;109(4):288-95.

20. Chalmers JD, Smith MP, McHugh BJ, Doherty C, Govan JR, Hill AT. Short- and long-term antibiotic treatment reduces airway and systemic inflammation in non-cystic fibrosis bronchiectasis. Am J Respir Crit Care Med. 2012;186(7):657-65. 
21. Ryan G, Singh M, Dwan K. Inhaled antibiotics for long-term therapy in cystic fibrosis. Cochrane Database Syst Rev. 2011;3: CD001021.

22. Gibson RL, Emerson J, McNamara S, Burns JL, Rosenfeld M, Yunker A, et al. Significant microbiological effect of inhaled tobramycin in young children with cystic fibrosis. Am J Respir Crit Care Med. 2003;167(6):841-9.

23. Valerius NH, Koch C, Høiby N. Prevention of chronic Pseudomonas aeruginosa colonisation in cystic fibrosis by early treatment. Lancet. 1991;338(8769):725-6.

24. McShane PJ, Naureckas ET, Tino G, Strek ME. Non-cystic fibrosis bronchiectasis. Am J Respir Crit Care Med. 2013;188(6):647-56.

25. Ratjen F, Rietschel E, Kasel D, Schwiertz R, Starke K, Beier H, et al. Pharmacokinetics of inhaled colistin in patients with cystic fibrosis. J Antimicrob Chemother. 2006;57(2):306-11.

26. Renée Crowther Labiris N, Holbrook AM, Chrystyn H, Macleod SM, Newhouse MT. Dry powder versus intravenous and nebulized gentamicin in cystic fibrosis and bronchiectasis. Am J Respir Crit Care Med. 1999;160(5):1711-6.

27. Yapa S, Li J, Patel K, Wilson JW, Dooley MJ, George J, et al. Pulmonary and systemic pharmacokinetics of inhaled and intravenous colistin methanesulfonate in cystic fibrosis patients: targeting advantage of inhalational administration. Antimicrob Agents Chemother. 2014;58(5):2570-9.

28. EUCAST. Clinical Breakpoints - bacteria (v 8.0) [Internet]. EUCAST; 2018 [cited 2018 May 2]. Available from: http://www. eucast.org/clinical_breakpoints/.

29. Weinstein, Melvin P. M100 Performance Standards for Antimicrobial Susceptibility Testing. 28th ed. CLSI; 296 p.

30. Finch S, McDonnell MJ, Abo-Leyah H, Aliberti S, Chalmers JDA. Comprehensive analysis of the impact of Pseudomonas aeruginosa colonization on prognosis in adult bronchiectasis. Ann Am Thorac Soc. 2015;12(11):1602-11.

31. Martinez-García MA. Pseudomonas aeruginosa infection and exacerbations in bronchiectasis: more questions than answers. Eur Respir J. 2018;51(2):1702497.

32. Loebinger MR, Wells AU, Hansell DM, Chinyanganya N, Devaraj A, Meister M, et al. Mortality in bronchiectasis: a long-term study assessing the factors influencing survival. Eur Respir J. 2009;34(4): 843-9.

33. Metersky ML, Aksamit TR, Barker A, Choate R, Daley CL, Daniels LA, et al. The prevalence and significance of Staphylococcus aureus in patients with non-cystic fibrosis bronchiectasis. Ann Am Thorac Soc. 2018:18.

34. King PT, Sharma R. The lung immune response to nontypeable Haemophilus influenzae (lung immunity to NTHi) [Internet]. Journal of Immunology Research. 2015 [cited 2018 Feb 5]. Available from: https://www.hindawi.com/journals/jir/2015/ 706376/.

35. Couch LA. Treatment with tobramycin solution for inhalation in bronchiectasis patients with Pseudomonas aeruginosa. Chest. 2001;120(3 Suppl):114S-7S.

36. Scheinberg P, Shore E. A pilot study of the safety and efficacy of tobramycin solution for inhalation in patients with severe bronchiectasis. Chest. 2005;127(4):1420-6.

37. Wilson CB, Jones PW, O'Leary CJ, Cole PJ, Wilson R. Validation of the St. George's Respiratory Questionnaire in bronchiectasis. Am J Respir Crit Care Med. 1997;156(2 Pt 1):536-41.

38. Orriols R, Roig J, Ferrer J, Sampol G, Rosell A, Ferrer A, et al. Inhaled antibiotic therapy in non-cystic fibrosis patients with bronchiectasis and chronic bronchial infection by Pseudomonas aeruginosa. Respir Med. 1999;93(7):476-80.

39. Barker AF, Couch L, Fiel SB, Gotfried MH, Ilowite J, Meyer KC, et al. Tobramycin solution for inhalation reduces sputum Pseudomonas aeruginosa density in bronchiectasis. Am J Respir Crit Care Med. 2000;162(2 Pt 1):481-5.
40. Drobnic ME, Suñé P, Montoro JB, Ferrer A, Orriols R. Inhaled tobramycin in non-cystic fibrosis patients with bronchiectasis and chronic bronchial infection with Pseudomonas aeruginosa. Ann Pharmacother. 2005;39(1):39-44.

41. Orriols R, Hernando R, Ferrer A, Terradas S, Montoro B. Eradication therapy against Pseudomonas aeruginosa in non-cystic fibrosis bronchiectasis. Respir Int Rev Thorac Dis. 2015;90(4):299-305.

42. Serisier DJ, Bilton D, De Soyza A, Thompson PJ, Kolbe J, Greville HW, et al. Inhaled, dual release liposomal ciprofloxacin in noncystic fibrosis bronchiectasis (ORBIT-2): a randomised, doubleblind, placebo-controlled trial. Thorax. 2013;68(9):812-7.

43. Wilson R, Welte T, Polverino E, De Soyza A, Greville H, O'Donnell A, et al. Ciprofloxacin dry powder for inhalation in non-cystic fibrosis bronchiectasis: a phase II randomised study. Eur Respir J. 2013;41 (5):1107-15.

44. De Soyza A, Aksamit T, Bandel T-J, Criollo M, Elborn JS, Operschall E, et al. RESPIRE 1: a phase III placebo-controlled randomised trial of ciprofloxacin dry powder for inhalation in non-cystic fibrosis bronchiectasis. Eur Respir J. 2018;51(1): 1702052. Respire I \& II are landmark multi-centre trials examining the role of inhaled dry-powder ciprofloxacin in the management of stable bronchiectasis.

45. Aksamit T, De Soyza A, Bandel T-J, Criollo M, Elborn JS, Operschall E, et al. RESPIRE 2: a phase III placebo-controlled randomised trial of ciprofloxacin dry powder for inhalation in non-cystic fibrosis bronchiectasis. Eur Respir J. 2018;51(1). Respire I \& II are landmark multi-centre trials examining the role of inhaled dry-powder ciprofloxacin in the management of stable bronchiectasis.): 1702053 .

46. Haworth CS, Foweraker JE, Wilkinson P, Kenyon RF, Bilton D. Inhaled colistin in patients with bronchiectasis and chronic Pseudomonas aeruginosa infection. Am J Respir Crit Care Med. 2014;189(8):975-82.

47. Murray MP, Govan JRW, Doherty CJ, Simpson AJ, Wilkinson TS, Chalmers JD, et al. A randomized controlled trial of nebulized gentamicin in non-cystic fibrosis bronchiectasis. Am J Respir Crit Care Med. 2011;183(4):491-9.

48. Barker AF, O'Donnell AE, Flume P, Thompson PJ, Ruzi JD, de Gracia J, et al. Aztreonam for inhalation solution in patients with non-cystic fibrosis bronchiectasis (AIR-BX1 and AIR-BX2): two randomised double-blind, placebo-controlled phase 3 trials. Lancet Respir Med. 2014;2(9):738-49.

49. McCoy KS, Quittner AL, Oermann CM, Gibson RL, RetschBogart GZ, Montgomery AB. Inhaled aztreonam lysine for chronic airway Pseudomonas aeruginosa in cystic fibrosis. Am J Respir Crit Care Med. 2008;178(9):921-8.

50. Oermann CM, Retsch-Bogart GZ, Quittner AL, Gibson RL, McCoy KS, Montgomery AB, et al. An 18-month study of the safety and efficacy of repeated courses of inhaled aztreonam lysine in cystic fibrosis. Pediatr Pulmonol. 2010;45(11):1121-34.

51. Retsch-Bogart GZ, Quittner AL, Gibson RL, Oermann CM, McCoy KS, Montgomery AB, et al. Efficacy and safety of inhaled aztreonam lysine for airway pseudomonas in cystic fibrosis. Chest. 2009; 135(5):1223-32.

52. Quittner AL, Marciel KK, Salathe MA, O'Donnell AE, Gotfried $\mathrm{MH}$, Ilowite JS, et al. A preliminary quality of life questionnairebronchiectasis: a patient-reported outcome measure for bronchiectasis. Chest. 2014;146(2):437-48.

53. Olveira C, Olveira G, Espildora F, Giron R-M, Muñoz G, Quittner $\mathrm{AL}$, et al. Validation of a quality of life questionnaire for bronchiectasis: psychometric analyses of the Spanish QOL-B-V3.0. Qual Life Res Int J Qual Life Asp Treat Care Rehabil. 2014;23(4):1279-92.

54. Quittner AL, O'Donnell AE, Salathe MA, Lewis SA, Li X, Montgomery AB, et al. Quality of life questionnaire-bronchiectasis: final psychometric analyses and determination of minimal important difference scores. Thorax. 2015;70(1):12-20. 